IAU Colloquium 164: Radio Emission from Galactic and Extragalactic Compact Sources

ASP Conference Series, Vol. 144, 1998

J. A. Zensus, G. B. Taylor, \&J.M. Wrobel (eds.)

\title{
VLBI of Southern EGRET Identifications
}

D. W. Murphy

Jet Propulsion Laboratory, Pasadena, CA 91109, U.S.A

S. J. Tingay, R. A. Preston, D. L. Meier \& D. L. Jones

Jet Propulsion Laboratory, Pasadena, CA 91109, U.S.A

P. G. Edwards

Institute of Space and Astronautical Science, Sagamihara, Kanagawa 229, Japan

M. E. Costa, J. E. J. Lovell \& P. M. McCulloch

University of Tasmania, Hobart, 7001, Australia

D. L. Jauncey, J. E. Reynolds, A. K. Tzioumis \& E. A. King

Australia Telescope National Facility, CSIRO, Epping, 2121, Australia

G. D. Nicolson \& J.F.H. Quick

Hartebeestoek Radio Astronomy Observatory, Krugersdorp, 1740, South Africa

T.-S. Wan \& Z.-Q. Shen

Shanghai Astronomical Observatory, Shanghai, 200030, People's Republic of China

Abstract. We have undertaken VLBI observations of 8 Southern Hemisphere EGRET radio sources. Using our data as well as data obtained from the literature we have examined the difference in radio properties between gamma-ray loud and gamma-ray quiet radio sources. In particular, we find no evidence that gamma-ray loud radio sources lie preferentially in sources with straight radio jets as has been suggested.

\section{Introduction}

All 42 identified EGRET sources are flat-spectrum radio sources, indicating that there is a strong relationship between gamma-ray and core radio emission. However, what is not clear is the exact nature of this relationship. Why are some strong core-dominated radio sources gamma-ray loud whereas others are not? In particular, we wanted to test the hypothesis, first suggested by von Montigny et al. (1995), that gamma-ray loud sources preferentially lie in straight jet sources. Under such a scenario, sources with straight jets pointing close to our line of sight, are both gamma-ray and radio loud. For bent jet sources the gamma-ray and radio beams are misaligned and the radio source is gamma-ray quiet.

\section{Observations}

We have undertaken VLBI observations of 8 EGRET sources with declinations < -10: PKS 0208-512, PKS 0521-365, PKS 0537-441, PKS 1127-145, PKS 1622253, PKS 1622-297, PKS 1730-130, and PKS 1908-201 with either the VLBA, at $8.4 \mathrm{GHz}$, or an array of radio telescopes in Australia and South Africa, at 4.8 $\mathrm{GHz}$ (Tingay et al. 1996).

In Figure 1, we show an example of one of our $8.4 \mathrm{GHz}$ VLBA images. 


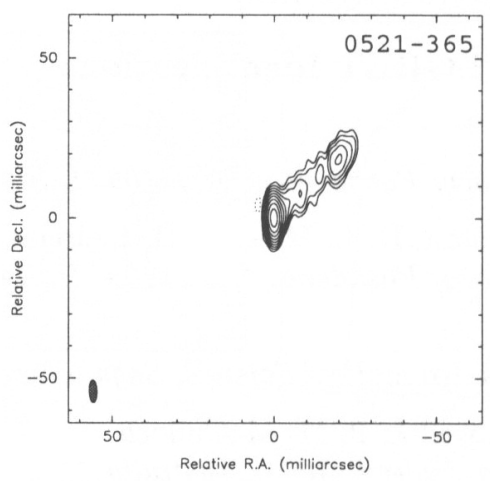

Figure 1. $8.4 \mathrm{GHz}$ VLBA image of the second lowest redshift EGRET source PKS 0521-365 $(\mathrm{z}=0.0554)$.

\section{Discussion}

We have combined our data with data in the literature to determine the radio properties of the 42 identified EGRET sources in the Mattox et al. (1997) sample. As a comparison sample of radio-loud but gamma-ray quiet sources we have used the 26 flat-spectrum gamma-ray quiet radio sources from the Pearson and Readhead survey (Pearson $\&$ Readhead 1988). We find no differences between the two samples in many properties: radio spectral index, redshift, misalignment between pc-scale and kpc-scale jets. Thus, we find no evidence that gamma-ray loud sources lie preferentially in sources with straight jets.

In our study we do however re-confirm that on average the EGRET sources have higher radio flux densities than the gamma-ray quiet radio sources (Impey 1996, Mattox et al. 1997).

Acknowledgments. Part of this work has been undertaken at the Jet Propulsion Laboratory, California Institute of Technology, under contract to the National Aeronautics and Space Administration. The National Radio Astronomy Observatory is a facility of the National Science Foundation, operated under a cooperative agreement by Associated Universities, Inc.The Australia Telescope is operated as a national facility by the CSIRO.

\section{References}

Impey, C. 1996. $A J, 112,2667-2683$.

Mattox, J. R., et al. 1997. ApJ, 481, 95-115.

Pearson, T. J., \& Readhead, A. C. S. 1988. ApJ, 328, 114-142.

Tingay, S. J., et al. 1996. $A p J, 464,170-176$.

von Montigny, C., et al. 1995. $A \& A, 299,680-688$. 